\title{
Genome-wide analysis of the CaHsp20 gene family in pepper: comprehensive sequence and expression profile analysis under heat stress
}

OPEN ACCESS

Edited by:

Jun Yu,

Beijing Institute of Genomics, China

Reviewed by:

$X u n X u$,

Beijing Genomics Institute, China

Jingfa Xiao,

Beijing Institute of Genomics, China

*Correspondence: Zhen-Hui Gong and Ming-Hui Lu, Department of Vegetable Science, College of Horticulture, Northwest A\&F University, No. 3 Taicheng Road,

Yangling, Shaanxi 712100, China zhgong@nwsuaf.edu.cn; xnjacklu@nwsuaf.edu.cn; Shu-Bin Wang Laboratory for Solanaceous Fruit Vegetable, Institute of Vegetable

Crops, Jiangsu Academy of Agricultural Sciences, No. 50

Zhongling Street, Nanjing, Jiangsu 210014, China wangsbpep@163.net

Specialty section:

This article was submitted to Plant Genetics and Genomics, a section of the journal Frontiers in Plant Science

Received: 04 June 2015 Accepted: 16 September 2015

Published: 01 October 2015

Citation:

Guo M, Liu J-H, Lu J-P, Zhai Y-F, Wang $H$, Gong $Z-H$, Wang $S-B$ and Lu M-H (2015) Genome-wide analysis of the CaHsp2O gene family in pepper: comprehensive sequence and expression profile analysis under heat stress. Front. Plant Sci. 6:806. doi: 10.3389/fpls.2015.00806

\author{
Meng Guo ${ }^{1}$, Jin-Hong Liu ${ }^{1}$, Jin-Ping L ${ }^{1}{ }^{1}$, Yu-Fei Zhai ${ }^{1}, H u$ Wang ${ }^{1}$, Zhen-Hui Gong ${ }^{1 *}$, \\ Shu-Bin Wang ${ }^{2 *}$ and Ming-Hui $\mathrm{Lu}^{1 *}$ \\ ${ }^{1}$ Department of Vegetable Science, College of Horticulture, Northwest A\&F University, Yangling, China, ${ }^{2}$ Laboratory for \\ Solanaceous Fruit Vegetable, Institute of Vegetable Crops, Jiangsu Academy of Agricultural Sciences, Nanjing, China
}

The Hsp20 genes are present in all plant species and play important roles in alleviating heat stress and enhancing plant thermotolerance by preventing the irreversible aggregation of denaturing proteins. However, very little is known about the CaHsp20 gene family in pepper (Capsicum annuum L.), an important vegetable crop with character of temperate but thermosensitive. In this study, a total of 35 putative pepper Hsp20 genes (CaHsp20s) were identified and renamed on the basis of their molecular weight, and then their gene structure, genome location, gene duplication, phylogenetic relationship, and interaction network were also analyzed. The expression patterns of CaHsp2O genes in four different tissues (root, stem, leaf, and flower) from the thermotolerant line R9 under heat stress condition were measured using semi-quantitative RT-PCR. The transcripts of most CaHsp2O genes maintained a low level in all of the four tissues under normal temperature condition, but were highly induced by heat stress, while the expression of CaHsp16.6b, 16.7, and 23.8 were only detected in specific tissues and were not so sensitive to heat stress like other CaHsp20 genes. In addition, compared to those in thermotolerant line R9, the expression peak of most CaHsp20 genes in thermosensitive line B6 under heat stress was hysteretic, and several CaHsp20 genes (CaHsp16.4, 18.2a, 18.7, 21.2, 22.0, 25.8, and 25.9) showed higher expression levels in both line $\mathrm{B} 6$ and R9. These data suggest that the CaHsp20 genes may be involved in heat stress and defense responses in pepper, which provides the basis for further functional analyses of $\mathrm{CaHsp20s}$ in the formation of pepper acquired thermotoleance.

Keywords: Capsicum annuum L., CaHsp20 genes, heat stress, expression profile, gene family

\section{Introduction}

As the sessile organisms, plants cannot escape the extreme environment conditions, including biotic and abiotic stresses, such as pests and pathogens, drought, salinity, cold, heat, and heavy metal stresses (Wang et al., 2003; Mittler, 2006; Mittler et al., 2012). Increase in the global warming having adverse effects of heat stress (HS) on crop plants growth, development and productivity 
are more serious, thus plants adaptive response to high temperature i.e., hypersensitive response (HSR) is very important for world food security (Glazebrook, 2001; Giorno et al., 2010). Under HS conditions, the heat shock transcription factors (Hsfs) bind to the heat shock elements (HSEs) of the promoters of heat-responsive genes, mainly the heat shock proteins (Hsps), to turn on or regulate their expression (Scharf et al., 2012; Waters, 2013). Plant Hsps as molecular chaperones are involved in maintaining homeostasis of protein folding, preventing or repairing the misfolding and degradation of proteins caused by HS, and then confer the acquisition of plant thermotolerance (Vierling, 1991; Sung et al., 2003; Charng et al., 2006).

The plant Hsps are grouped into five families based on the molecular weight and sequence homology, including the Hsp100s, Hsp90s, Hsp70s, Hsp60s, and Hsp20s (Sarkar et al., 2009; Waters, 2013). Among all the five conserved families, Hsp20s, a group of small heat shock proteins (sHsps) with molecular size ranging from 15 to $42 \mathrm{kDa}$, are the most prevalent and abundant family induced by HS in many higher plants. Furthermore, they can function as molecular chaperone without ATP to maintain the stability of proteins, which suggest that Hsp20s may play a vital role during the formation of plant acquired thermotolerance (Vierling, 1991; Sun et al., 2002; Wang et al., 2004; Waters, 2013; Haslbeck and Vierling, 2015).

Hsp20s share a conserved modular structure, and the most striking feature is a core conserved alpha-crystallin domain (ACD, the Hsp20 domain) containing 90 amino acids flanked by a variable $\mathrm{N}$-terminal domain and a C-terminal extension (Vierling, 1991; Scharf et al., 2001; Waters, 2013), in which the $\mathrm{ACD}$ is involved in substrate interactions, the $\mathrm{N}$-terminus is responsible for substrate binding and the $\mathrm{C}$-terminal participates in homo-oligomerization and the formation of HS granula (Kirschner et al., 2000; Giese and Vierling, 2004; Basha et al., 2006; Jaya et al., 2009). The ACD is composed of two anti-parallel sheets of four and three $\beta$-strands, i.e., conserved regions I (CRI, $\beta 2-\beta 3-\beta 4-\beta 5)$ in $\mathrm{N}$-terminal consensus and CRII $(\beta 7-\beta 8-\beta 9)$ in $\mathrm{C}$-terminal consensus, respectively, connected by a hydrophobic region loop ( $\beta 6$-loop; Bondino et al., 2012; Haslbeck and Vierling, 2015). Besides, the $\mathrm{N}$-terminus contains the transit, targeting or signal sequences, and the C-terminal may also contain organellespecific retention amino acid motifs (Waters, 2013).

The Hsp20s form a more diverse family than other four Hsp families in plants, and are also far more than the members of Hsp20 families in animals (Wang et al., 2004; Waters, 2013). Plant Hsp20s are encoded by nuclear genes and are divided into various subfamilies based on cellular location, sequence homology and function (Vierling, 1991; Waters et al., 1996). Nineteen Hsp20 genes in Arabidopsis (Scharf et al., 2001; Waters, 2013), 23 in Oryza sativa, 36 in Populus trichocarpa (Waters et al., 2008), and 51 in Glycine max were identified, furthermore, the Hsp20 genes in Arabidopsis were grouped into 11 subfamilies (Scharf et al., 2001; Waters, 2013), while those from G. max were grouped into 15 subfamilies based on phylogenetic analysis, prediction of subcellular localization and prediction of protein secondary structure models (Lopes-Caitar et al., 2013). However, to our knowledge, genome-wide analysis of Hsp20 family in pepper is still lacking.

Pepper (Capsicum annuum L.) is an important vegetable crop worldwide, and is deeply loved by a large population due to its major ingredient in cuisines, essential vitamins and other healthy nutrients (Kim et al., 2014). However, pepper is sensitive to high temperature, especially in reproductive stage (Guo et al., 2014). Once suffering continuous HS, the pollination and fertilization of pepper will be severely damaged, which can greatly reduce the yield and quality of pepper fruits. Therefore, study of Hsp20s is important for understanding the mechanism of heat response in pepper, while except several CaHsp20 genes, such as CaHsp24 (Zhu et al., 2011), CaHsp26 (Guo et al., 2007), little is currently known about the C. annuum heat shock protein 20 (CaHsp20) family.

In this study, we identify and analyze $35 \mathrm{CaHsp} 20$ genes from pepper genome, focusing on their heat-induced expression in various tissues, and their dynamic expression patterns in two different thermotolerant pepper lines under HS condition. Our results provide a foundation for further functional analysis of CaHsp20 members, and will be valuable for illuminating the mechanism of pepper acquired thermotolerance, and even the adaptability of pepper to other stress conditions.

\section{Materials and Methods}

\section{Genome-wide Identification of CaHsp20 Genes in Pepper}

The Hsp20 proteins are defined by possessing a highly conserved 80-100 amino acid residues called ACD, and a low molecular weight (15-42 kDa; Vierling, 1991; Scharf et al., 2001). To identify the Hsp20 family members in pepper, the Hidden Markov Model (HMM) profile of ACD (PF00011) downloaded from the Pfam protein family database (http://pfam.sanger.ac.uk) was used as queries to search the Pepper Genome Database (PGD, http:// peppergenome.snu.ac.kr/, CM334, Zunla-1 and glabriusculum genomes; Kim et al., 2014; Qin et al., 2014). Secondly, all $O$. sativa and G. max Hsp20 protein sequences (Ouyang et al., 2009; Lopes-Caitar et al., 2013) were used as query sequences to search against the PGD and the NCBI database. All output putative pepper $H s p 20$ genes with an $e$-value $\leq 0.001$ were assembled to remove redundancy, and then submitted to Pfam and SMART (http://smart.embl-heidelberg.de/) to confirm the presence of the ACD domain. The Multiple Sequence Alignment (MSA) was subsequently trimmed in order to remove subsequences that were not truly homologous. The predicted genes lacking an ACD domain and/or the genes whose molecular weight out of the range from 15 to $42 \mathrm{kDa}$ were rejected. All nonredundant and confident genes were assigned as pepper Hsp20 genes (CaHsp20s), and annotated based on their molecular weight as with soybean (Lopes-Caitar et al., 2013). The logic flowchart of identification of pepper Hsp20 genes was shown in Figure S1.

Using the same method, the putative Hsp20 homologs in tomato, Arabidopsis, rice, and maize were obtained from the SOL 
Genomics Network (SGN) database (http://solgenomics.net/ organism/Solanum_lycopersicum/genome), the TAIR database (https://www.arabidopsis.org/index.jsp), the Rice Genome Annotation Project (http://rice.plantbiology.msu.edu/), and the TIGR Maize Database (http://maize.jcvi.org/), respectively.

The protein sequences of putative CaHsp20 members were analyzed with EXPASY PROTOPARAM (http://www.expasy. org/tools/protparam.html) to obtain the number of amino acids, molecular weight, theoretical isoelectric point (pI) and instability index (with a value $>40$ considered as unstable; Lopes-Caitar et al., 2013). The chromosomal location data and intron numbers of putative CaHsp20s were retrieved from PGD. The WoLF PSORT program (http://wolfpsort.org/) and TargetP (Emanuelsson et al., 2000) were used for predicting the subcellular locations.

\section{Analysis of Conserved Motifs of Pepper CaHsp2O Family}

The conserved domains of the CaHsp20 proteins (ACD) were confirmed by Pfam and SMART. The MEME program (Version 4.10.0, http://meme.nbcr.net/meme/tools/meme) was used for identification of the conserved motifs, with the following parameters: number of repetitions-any, maximum number of motifs-10, the optimum motif widths set from 6 to 200 amino acid residues; the conserved motifs were annotated with InterProScan (http://www.ebi.ac.uk/interpro/search/sequencesearch; Ouyang et al., 2009). The ACD sequences of all CaHsp20 members were aligned using the Promals3D structural alignment program (http://prodata.swmed.edu/promals3d/promals3d. php).

\section{Phylogenetic Analysis and Classification of Pepper CaHsp20 Genes}

The full amino acid sequences of Hsp20 members from pepper, tomato, Arabidopsis, rice, and maize were aligned by CLUSTALW program. The gene IDs of Hsp20 members from tomato, Arabidopsis, rice, and maize were shown in Table S1. An unrooted neighbor-joining phylogenetic tree was constructed using MEGA 5.10 software (Tamura et al., 2011) with the bootstrap test replicated 1000 times, pair wise deletion and a Poisson model. The pepper CaHsp20 genes were assigned to different groups based on the multiple sequence alignment and the classification of Hsp20 genes in the other four species.

\section{Chromosomal Localization and Gene Duplication}

Each of the CaHsp20 genes was mapped on chromosomes using MapDraw (Liu and Meng, 2003) based on their chromosomal position and the relative distance between these genes on the same chromosome derived from PGD. The CaHsp20 gene duplication was defined based on the following criteria: (1) the length of the sequence alignment covered $\geq 70 \%$ of the longer gene; and (2) the similarity of the aligned gene regions $\geq 70 \%$ (Gu et al., 2002; Yang et al., 2008). Genes separated by five or fewer genes in a $100-\mathrm{kb}$ region were considered to be tandem duplicates (Wang et al., 2010); genes were regarded as segmental duplication if they were found to be coparalogs located on duplicated chromosomal blocks, as proposed by Wei et al. (2007).

\section{Prediction of CaHsp20s Protein-Protein Interaction Network}

An interaction network of CaHsp20 proteins from pepper was constructed to understand the genome-wide regulation network; the interolog from Arabidopsis was used for predicting protein-protein interaction network of CaHsp20 members. An interaction work of Arabidopsis Hsp20 proteins was constructed using the Arabidopsis Interactions Viewer (http://bar.utoronto.ca/interactions/cgi-bin/arabidopsis_interac tions_viewer.cgi). Arabidopsis Hsp20 proteins were mapped to pepper CaHsp20 proteins based on their homologous relationship, and an interaction network of CaHsp20 proteins was drawn by Cytoscape_v2.8.3 (National Institute of General Medical Sciences, MD, USA).

\section{Plant Materials and Heat Stress Treatments}

The pepper thermotolerant line R9 and the thermosensitive line B6 were used in this study. Both lines were grown in a growth chamber at $26^{\circ} \mathrm{C}$ (day for $16 \mathrm{~h}$ ) $/ 20^{\circ} \mathrm{C}$ (night for $8 \mathrm{~h}$ ) till reaching the age of 6-8 true leaves. R9 plants were used for the tissue specific expression analysis, the seedlings with 6-8 true leaves and the plants with flower buds were treated with $\mathrm{HS}$ of $40^{\circ} \mathrm{C}$ for $2 \mathrm{~h}$ by placing in a light incubator (GXZ-380C, Jiangnan Instrument Factory, Ningbo, China) and others under $26^{\circ} \mathrm{C}$ for $2 \mathrm{~h}$ were used as the control. The young leaves, stems and roots from seedling plants and the flower buds from adult plants with or without HS treatments were collected. For the dynamic expression analysis of CaHsp20s under HS, after being subjected to $40^{\circ} \mathrm{C}$ for $0,0.5$, $1,2,4 \mathrm{~h}$, the leaves in B6 and $\mathrm{R} 9$ were collected, respectively. Temperature treatment was initiated during the light period and all the samples were harvested within the light period. These collected samples were frozen with liquid nitrogen for total RNA extraction and cDNA synthesis. Each treatment was conducted with three biological replicates, and samples from five plants were collected for each replicate.

\section{RNA Isolation and Expression Analysis of Pepper CaHsp20 Genes}

Total RNA was extracted using Total RNA kit (BioTeke, Beijing, China) and reverse-transcribed using SuperScriptIII Reverse Transcriptase (Takara, Dalian, China), the operational procedure followed the manufacturer's procedure, and the cDNA was diluted to $50 \mathrm{ng} / \mu \mathrm{L}$ with $\mathrm{ddH}_{2} \mathrm{O}$ for further examination. For expression analysis of pepper CaHsp20s in different tissues, semi-quantitative reverse-transcription (RT) PCR reactions were employed. Primer pairs (Table S2) were designed by Primer Premier 5.0 and tested by NCBI Primer BLAST. Ubiquitin binding protein gene $C a U B I-3$ was used as the reference genes (Wan et al., 2011). PCR reactions for all genes were conducted with the following program: initial denaturation at $94^{\circ} \mathrm{C}$ for $5 \mathrm{~min}$, followed with denaturation at $94^{\circ} \mathrm{C}$ for $30 \mathrm{~s}$, annealing at $52^{\circ} \mathrm{C}$ for $30 \mathrm{~s}$, extension at $72^{\circ} \mathrm{C}$ for $30 \mathrm{~s}$ with 30 cycles, and final extension at $72^{\circ} \mathrm{C}$ for $10 \mathrm{~min}$. PCR products were detected by agarose gel electrophoresis with $1.5 \%$ gel concentration. The expression levels of CaHsp20 genes in each sample were compared with the internal control gene, CaUBI-3. 
Real-time quantitative PCR (qRT-PCR) was conducted using SYBR Green Supermix (Takara, Dalian, China) with a total volume of $20 \mu \mathrm{L}$ reaction system on the iQ5.0 Bio-Rad iCycler thermocycler (Bio-Rad, Hercules, CA, USA). The primers of CaHsp20 genes were the same as those used for semi-quantitative RT-PCR. The qRT-PCR reactions were performed as described (Guo et al., 2014). The CaUBI-3 gene was used as the internal reference gene. Three independent biological replicates were carried out and qRT-PCR of each replicate was performed in triplicate. The relative expression levels of pepper $H s p 20$ genes were calculated as $2^{-\Delta \Delta C T}$ method (Schmittgen and Livak, 2008).

\section{Search for cis-acting Elements in the Promoters of Pepper CaHsp20 Genes}

The upstream regions $(0.5 \mathrm{~kb})$ of the CaHsp20 genes were derived from PGD, and were searched for regulatory elements, including HSEs (cis-acting element involved in heat stress responsiveness), TATA-box (core promoter element around -30 of transcription start), TC-rich repeats (cis-acting element involved in defense and stress responsiveness), W-box (binding site for the WRKY transcription factor in defense response), and CCAAT sequences (enhancer-binding protein factors) in the promoters were performed in PlantCARE (http://bioinformatics. psb.ugent.be/webtools/plantcare/html/; Lescot et al., 2002) and PLACE database (Higo et al., 1999).

\section{Results}

\section{Identification and Analysis of CaHsp20 Gene Family Members in Pepper}

A total of 41 sequences of $H s p 20$ gene were gotten from PGD by HMM analysis, out of which 35 were annotated as being pepper Hsp20 genes based on the confirmation of Pfam and SMART scans, and sequences without a complete domain (CA09g15370, $C A 12 g 15460$, and CA03g18690) and sequences whose molecular weight out of the range of Hsp20s (CA08g09970, CA04g23450, and CA00g46140) were removed. The $35 \mathrm{CaHsp20}$ genes were named according to their molecular weight (Table 1).

The length of CaHsp20 proteins ranged from 141 (CaHsp16.4) to 313 amino acids (CaHsp35.5), and the predicted molecular weights of the CaHsp20s were between $16.33 \mathrm{kDa}$ (CaHsp16.3) and $35.53 \mathrm{kDa}$ (CaHsp35.5). The CaHsp20s shared a conserved ACD comprised about 90 amino acids, in which CaHsp24.3b owned the shortest ACD with 74 amino acids, while CaHsp16.3's ACD (116 amino acids) was the longest. The predicted pI-values of CaHsp20 proteins ranged from 5.07 (CaHsp18.4a) to 9.52 (CaHsp26.5), and the predicted instability indexes showed that only nine of the $35 \mathrm{CaHsp} 20$ s were stable proteins (instability index $=40)$. In addition, it was also found that $16(45.71 \%)$ of the total 35 CaHsp20 genes had no introns, 18 genes (51.43\%) had a single intron, while only CaHsp27.6 had five introns.

\section{Characterization of the Deduced Amino Acid Sequences of CaHsp20s}

Ten consensus motifs were found in CaHsp20 proteins by MEME motif search tool (Figure 1; Table 2). Based on the analysis of
Pfam, SMART, and InterProScan, the full sequences of motif 1, 2, 3, and 4 formed the highly conserved ACD (Figure 2A), in which the combined sequence of motif 2 and 4 contained the CRI of ACD, while the combined sequence of motif 3 and 1 contained the $\beta 6$-loop and CRII. The four motifs were detected in majority of the CaHsp20 proteins, while only motif 2 was found in CaHsp16.7 and CaHsp30.1, and CaHsp16.6b did not contain motif 2. Motif 6 was detected in CaHsp22.1, 23.8, and 26.5, and motif 8 was found behind the motif 5 in CaHsp18.1a, 18.1b, 18.2a, and $18.2 \mathrm{~b}$, while motif 10 were only found in the front of motif 2 in CaHsp17.7a, 17.7b, and 25.8 (Figure 1). Only motif 6 was found in the C-terminal regions, other motifs such as motif 5 , $7,8,9$, and 10 were found mainly in the N-terminal regions. It was worth noting that motif 9 (the signal-peptide sequence) was found in CaHsp18.7, 21.1, 21.2, 22.0, and 22.6, which was consistent with their prediction of secretory pathway localization (Table 1).

The ACD was formed by two conserved regions, CRI (motif 2 and 4 ) with $\beta 2, \beta 3, \beta 4$, and $\beta 5$ and CRII (part of motif 3 and 1) with $\beta 7, \beta 8$, and $\beta 9$, which were separated by a hydrophilic domain $\beta 6$-loop (part of motif 3 ) with variable length. The multiple alignments of the conserved ACD domains, spanning $\sim 90$ amino acids in CaHsp20 proteins was clearly shown in Figure 2B. CaHsp16.6b, 16.7, 24.3b, 27.6, 28.4, 30.1, and 35.5 either lacked the $\beta 6$-loop or contained sequences with unknown function between $\beta 5$ and $\beta 7$. The shorter ACDs of CaHsp18.4b, 24.3b, and 28.4 lacked the $\beta 2$ (Figure 2B; Table 1), which might show the functional diversity and remains to be fully elucidated.

\section{Phylogenetic Analysis of CaHsp20 Family}

To analyze the evolutionary relationship of the Hsp20 gene families in pepper, tomato, Arabidopsis, rice, and maize, the amino acid sequences of $154 \mathrm{Hsp} 20$ proteins, including 35 from pepper, 24 from tomato, 25 from Arabidopsis, 35 from rice, and 35 from maize were aligned and used to construct an unrooted phylogenetic tree. As shown in Figure 3, $130 \mathrm{Hsp} 20 \mathrm{~s}$ could be grouped into 12 distinct subfamilies, containing $47 \mathrm{CIs}$ (cytosol/nucleus I), 27 CIIs, 6 CIIIs, 1 CIVs, 5 CVs, 3 CVIs, 4 CVIIs, 5 MIs (mitochondria I), 4 MIIs, 19 Ps (plastid), 4 ERs (endoplasmic reticulum), and 5 Pos (peroxisomal), respectively. However, the left 24 proteins from pepper, Arabidopsis, rice and maize were not well-clustered into any subfamily. The Hsp20 members from the five species were more closely to those in the same class in different species than to others in the same species. Many Hsp20s from the five species were clustered into class CI and CII, including 9 and 10 CaHsp20 proteins, respectively, which indicated that cytosol might be the main function area for CaHsp20s. The CI subfamily had two subclades that consisted of monocotyledon Hsp20s (OsHsp20s and $\mathrm{ZmHsp20s}$ ) and two subclades that consisted of dicotyledon Hsp20s (CaHsp20s, SlHsp20s, and AtHsp20s), suggesting a single gene duplication took place prior to monocotyledondicotyledon bifurcation (Bondino et al., 2012). It was interesting that the members of $\mathrm{P}$ and $\mathrm{M}$ (MI and MII) subfamilies were closely related to each other, which was consistent with the Hsp20 of M subfamily evolved later from the $\mathrm{P}$ subfamily 
TABLE 1 | The list of CaHsp20 members identified.

\begin{tabular}{|c|c|c|c|c|c|c|c|c|c|c|c|}
\hline No. & Name & Gene ID & Chr. & ORF & Introns & AA & ACD & WT & pl & Localization predicted & Instability index \\
\hline 1 & CaHsp16.3 & CA05g01890 & 5 & 444 & 1 & 147 & $29-144$ & 16.33 & 6.84 & cyto, mito & 47.58 \\
\hline 2 & CaHsp16.4 & CA02g30320 & 2 & 426 & 1 & 141 & $29-138$ & 16.35 & 8.47 & Secretory pathway & 33.80 \\
\hline 3 & CaHsp16.6a & CA03g37070 & 3 & 447 & 1 & 148 & $40-145$ & 16.55 & 5.79 & cyto, extr & 65.53 \\
\hline 4 & CaHsp16.6b & CA10g22470 & 10 & 435 & 1 & 144 & $16-97$ & 16.55 & 9.04 & cyto, extr, E.R. & 14.10 \\
\hline 5 & CaHsp16.7 & CA04g23370 & 4 & 462 & 1 & 153 & $35-130$ & 16.66 & 6.29 & chlo, extr & 47.17 \\
\hline 6 & CaHsp17.2 & CA09g08950 & 9 & 447 & 0 & 148 & $38-147$ & 17.22 & 5.66 & cyto & 37.04 \\
\hline 7 & CaHsp17.6 & Capana06g000385 « & 6 & 465 & 0 & 154 & $50-153$ & 17.60 & 5.83 & cyto & 49.55 \\
\hline 8 & CaHsp17.7a & Capana03g001238॥ & 3 & 477 & 0 & 158 & $52-156$ & 17.73 & 6.17 & cyto, nucl & 39.33 \\
\hline 9 & CaHsp17.7b & Capana03g001241 « & 3 & 477 & 0 & 158 & $52-156$ & 17.68 & 6.75 & cyto, nucl & 36.08 \\
\hline 10 & CaHsp17.8a & CA06g24940 & 6 & 465 & 0 & 154 & $50-153$ & 17.76 & 5.83 & cyto & 52.77 \\
\hline 11 & CaHsp17.8b & CA06g24930 & 6 & 465 & 0 & 154 & $50-153$ & 17.78 & 6.00 & cyto & 53.63 \\
\hline 12 & CaHsp18.1a & CA08g17060 & 8 & 480 & 0 & 159 & $55-158$ & 18.12 & 5.58 & cyto & 68.08 \\
\hline 13 & CaHsp18.1b & CA08g17070 & 8 & 480 & 0 & 159 & $55-158$ & 18.09 & 5.84 & cyto & 71.86 \\
\hline 14 & CaHsp18.2a & CA08g17040 & 8 & 480 & 0 & 159 & $55-158$ & 18.22 & 5.39 & cyto, nucl & 69.52 \\
\hline 15 & CaHsp18.2b & CA08g17050 & 8 & 480 & 0 & 159 & $55-157$ & 18.18 & 8.83 & cyto & 58.82 \\
\hline 16 & CaHsp18.4a & Capana00g002751^ & 4 & 510 & 1 & 169 & $64-168$ & 18.43 & 5.07 & cyto & 56.93 \\
\hline 17 & CaHsp18.4b & Capana01g000571^ & 1 & 513 & 1 & 170 & $89-163$ & 18.43 & 5.68 & chlo & 37.86 \\
\hline 18 & CaHsp18.7 & CA06g20260 & 6 & 486 & 1 & 161 & $66-160$ & 18.73 & 5.08 & Secretory pathway & 58.55 \\
\hline 19 & CaHsp21.1 & Capana06g000831^ & 6 & 561 & 0 & 187 & $71-168$ & 21.12 & 6.36 & Secretory pathway & 42.46 \\
\hline 20 & CaHsp21.2 & Capang01g004332 ^ & 8 & 558 & 0 & 186 & $72-167$ & 21.20 & 5.20 & Secretory pathway & 46.21 \\
\hline 21 & $\mathrm{CaHsp} 21.7$ & CA07g18820 & 7 & 573 & 1 & 190 & $89-185$ & 21.67 & 5.19 & cyto, pero & 38.57 \\
\hline 22 & CaHsp22.0 & CA03g27140 & 3 & 579 & 0 & 192 & $70-173$ & 22.03 & 6.05 & Secretory pathway & 48.98 \\
\hline 23 & CaHsp22.1 & 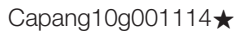 & 10 & 585 & 1 & 194 & $96-187$ & 22.11 & 9.14 & chlo & 45.05 \\
\hline 24 & CaHsp22.6 & CA05g03530 & 5 & 603 & 0 & 200 & $78-186$ & 22.63 & 5.37 & Secretory pathway & 38.03 \\
\hline 25 & CaHsp23.8 & CA09g06120 & 9 & 612 & 1 & 203 & $102-203$ & 23.83 & 7.15 & cyto, nucl & 54.99 \\
\hline 26 & CaHsp24.2 & CA01g05030 & 1 & 636 & 1 & 211 & $115-210$ & 24.15 & 5.18 & cyto, chlo, mito & 61.58 \\
\hline 27 & CaHsp24.3a & CA09g08090 & 9 & 648 & 0 & 215 & $119-214$ & 24.27 & 7.86 & chlo, cyto, mito & 68.39 \\
\hline 28 & CaHsp24.3b & Capana01g001839^ & 1 & 648 & 1 & 215 & $35-108$ & 24.32 & 9.21 & cyto, golg, nucl & 42.06 \\
\hline 29 & CaHsp25.8 & CA03g21390 & 3 & 705 & 1 & 234 & $135-233$ & 25.78 & 6.53 & chlo & 40.04 \\
\hline 30 & CaHsp25.9 & CA01g11490 & 1 & 690 & 1 & 229 & $128-228$ & 25.93 & 8.36 & chlo, mito & 40.47 \\
\hline 31 & CaHsp26.5 & CA10g10840 & 10 & 687 & 0 & 228 & $128-227$ & 26.49 & 9.52 & mito & 50.30 \\
\hline 32 & CaHsp27.6 & CA07g15940 & 7 & 756 & 5 & 251 & $156-250$ & 27.63 & 8.99 & chlo, nucl & 54.52 \\
\hline 33 & CaHsp28.4 & CA11g15400 & 11 & 765 & 1 & 254 & $30-109$ & 28.35 & 8.35 & golg, nucl, cyto & 40.24 \\
\hline 34 & CaHsp30.1 & Capana12g001708^ & 12 & 804 & 1 & 267 & $35-127$ & 30.14 & 8.26 & Secretory pathway & 47.98 \\
\hline 35 & CaHsp35.5 & CA04g13680 & 4 & 942 & 1 & 313 & $35-129$ & 35.53 & 7.70 & cyto, nucl & 33.82 \\
\hline
\end{tabular}

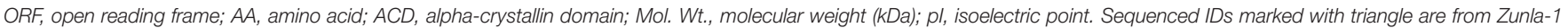
genome, with pentagram are from glabriusculum genome, others are from CM334 genome.

(Waters, 2013). It was noteworthy that no Hsp20 proteins from monocotyledons (rice and maize) were clustered into CIV cluster, which was consistent with the result suggested by Siddique et al. (2008) that CIV members of Hsp20 proteins were exclusively found in dicotyledonous. For the three species of dicotyledon, 12 pairs of orthologs (11 pairs of pepper/tomato orthologs and one pair of pepper/Arabidopsis otholog) were identified, indicating that the ancestral genes of Hsp20 family might have existed before differentiation of Arabidopsis, tomato and pepper species. In addition, several pairs of paralog were found in all of the three Hsp20 families from pepper, Arabidopsis and tomato, suggesting that CaHsp20 genes underwent many rounds of duplication events occurred after the pepper speciation.
It was found that the phylogenetic classification of Hsp20 family was consistent with the pattern of intron type (pattern 1 had no intron, pattern 2 had one intron, and pattern 3 had more than one intron) in previous report (Ouyang et al., 2009). The most CaHsp20 members of CI and ER subfamilies had no intron, and the members of CIII, CVII, P, and MII showed one intron (Figure 3; Table 1). However, six members belonged to CII subfamily showed no intron, other four members of CII subfamily had one intron. It was noteworthy that CaHsp16.3 belonged to Po subfamily had one intron (pattern 2), which was different from the Po subfamily from rice (pattern 1; Ouyang et al., 2009). Among the unclassified CaHsp20s, CaHsp27.6 was an orphan gene with five introns (pattern 3), which indicated particular phylogenetic status. 


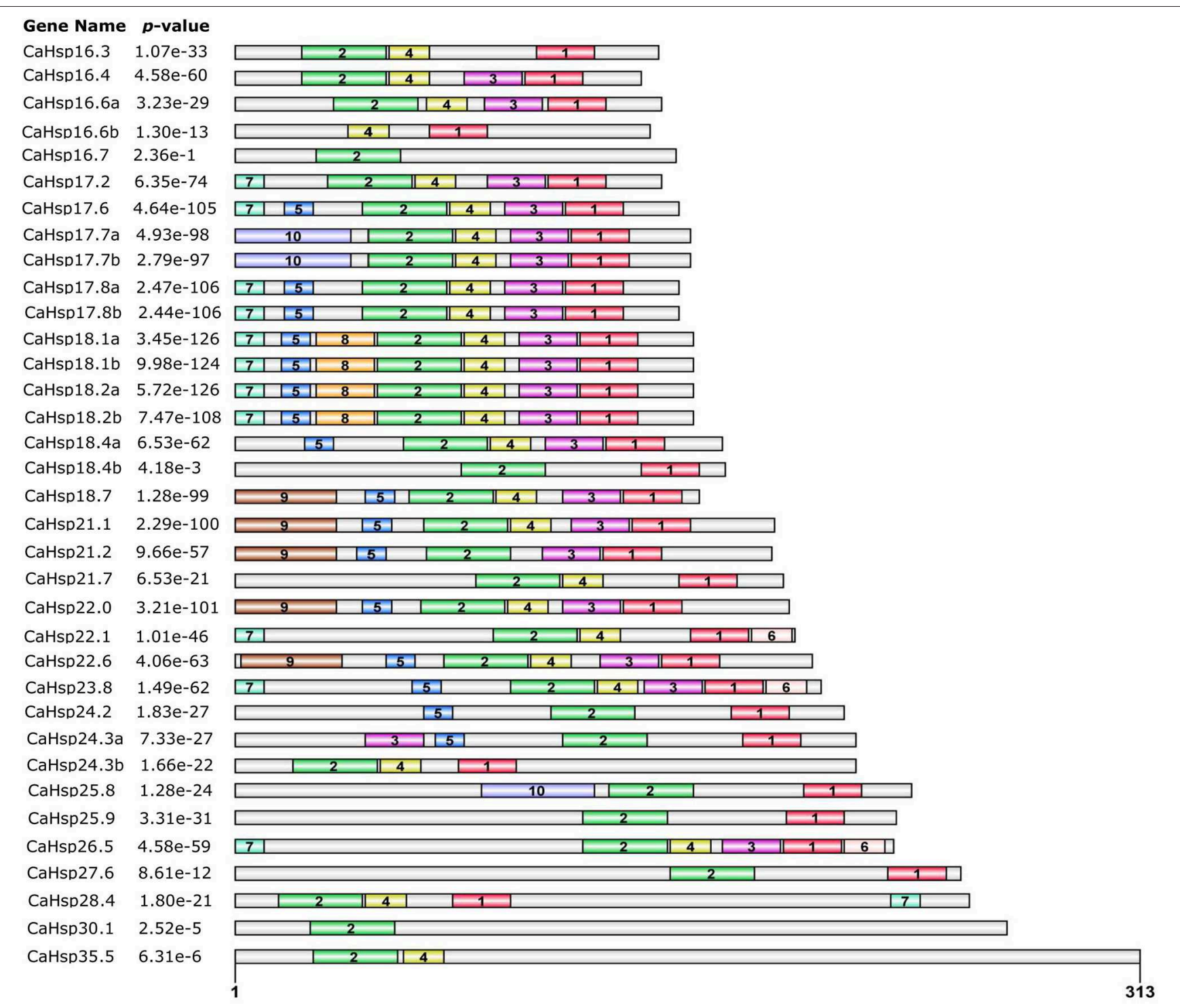

FIGURE 1 | Distribution of conserved motifs in the CaHsp20 family members. Ten putative motifs are represented by a number in colored box. Names of all members and combined $p$-values are shown on the left side of the figure and motif sizes are indicated at the bottom of the figure. For details of motifs refer to Table 2.

\section{Chromosomal Localization and Gene Duplication of CaHsp20 Genes}

The 35 CaHsp20 genes were distributed on all 12 pepper chromosomes (Figure 4; Table 1). Among these 35 CaHsp20 genes, five (14.3\%) genes were located on each of chromosome 3 , 6 , and 8 , four genes $(11.4 \%)$ on chromosome 1 , three genes on each of chromosome $4,9,10$, two genes on each of chromosome 5 and 7, and only one gene on each of chromosome 2, 11, and 12. As gene duplication events involve in the evolution of plant genomes, the duplications of CaHsp20s were also analyzed. As shown in Figure 4, one segmental duplication event was detected between CaHsp24.2 on chromosome 1 and CaHsp24.3a on chromosome 9, and three tandem duplication events (CaHsp17.7a/17.7b, CaHsp17.6/17.8a/17.8b and CaHsp18.1a/18.1b/18.2a/18.2b) were identified on chromosome 3, 6, and 8, respectively. However, CaHsp18.7, 21.1 , and 22.0 were likely to originate from the same ancestor gene, which underwent one segmental duplication and one tandem duplication event during the pepper chromosome evolution. These results suggested that both tandem and segmental duplication events made an important contribution to pepper CaHsp20 genes expansion.

\section{The Interaction Network of CaHsp20 Genes}

To further analyze the relationship between CaHsp20 genes and other genes, an interaction network of CaHsp20 genes was built according to the orthologous to Arabidopsis (Figure 5). There were $24(68.6 \%)$ out of 35 CaHsp20 genes orthologously related to $13(68.4 \%)$ out of 19 Arabidopsis Hsp20 genes. The red, blue, and green lines stand for Pearson correlation coefficients 
TABLE 2 | Motif sequences identified by MEME tools.

\begin{tabular}{lcl}
\hline Motif & Length (aa) & Sequence \\
\hline 1 & 21 & LPENADMDQIKAHMENGVLTV \\
2 & 30 & ANARWDWKETPEAHIFKADMPGYKKEDVKV \\
3 & 21 & KKNDQWHRMERSSGKFWRRFR \\
4 & 15 & QVEEGRVLQISGERK \\
5 & 11 & DPFSMDLWDPF \\
6 & 15 & TVPKEEVKKPDVKAI \\
7 & 11 & MSLIPSFFGGR \\
8 & 21 & GFPISSTIANTPSSARETSAF \\
9 & 36 & MVKATVSLISFLVLAIVMAFLPSKNEALIPYTRPTW \\
10 & 41 & MDFRLMGMDNTPIFHVLQHMMDTAGDESDKSVNAPS \\
& & RNYVR
\end{tabular}

Motif numbers corresponded to the motifs in Figure 1

$>0,<0$, and not calculated, which were corresponding to positive correlation (136 pairs of interacting genes), negative correlation (99 pairs), and unclear correlation (106 pairs), respectively. The interaction network of CaHsp20 genes showed a complex functional relationship. For example CaHsp24.2 directly or indirectly interacted with many other CaHsp20 genes (CaHsp17.8a, 17.8b, 22.1, 23.8, 26.5, 18.1a, 18.1b, 18.2a, and $18.2 b$ ), and also had more interacted genes than other CaHsp20s, while only one gene (CA03g01490) interacted with CaHsp25.8. In addition, CaHsp18.1a, 18.1b, 18.2a, and 18.2b genes interacted with heat stress related genes (heat shock protein 70 family genes CA03g30260 and CA01g31330), CaHsp17.7a and $17.7 b$ genes interacted with heavy metal stress related gene (heavy metal transport/detoxification superfamily protein gene CA02g24070), CaHsp16.3 interacted with signal transduction related gene (calcium-binding EF-hand family protein gene CA10g18540), and CaHsp25.9 with transcription factor (MADSbox transcription factor family protein gene CA00g74550), which might indicate that $\mathrm{CaHsp} 20$ genes involved in many response mechanisms by regulating their downstream genes or being regulated by their upstream regulators in pepper.

\section{Expression Patterns of CaHsp20 Genes in Different Tissues}

To primarily investigate the biological functions of individual CaHsp20 members in pepper, the heat-induced expression of these CaHsp20 genes were examined by semi-quantitative RTPCR in four major tissues (root, stem, leaf, and flower) from thermotolerant line R9 (Figure 6). Under normal temperature condition, 27 of the 35 CaHsp20 genes showed no significant tissue specificity as they were barely expressed in the tested tissues, however, the left eight CaHsp20 genes, including CaHsp16.3, 16.6b, 16.7, $17.7 a, 17.7 b, 18.1 b, 18.2 a$, and 23.8 could be detected at least in one tissue. Twenty-two (62.9\%) CaHsp20 genes were highly induced in at least one tissue after HS treatment $\left(40^{\circ} \mathrm{C}\right.$ for $\left.2 \mathrm{~h}\right)$. For example CaHsp16.3, 16.4, 17.6, and $18.1 b$ were expressed at relatively high levels in all of tissues, while CaHsp16.7 and 23.8 were highly induced by HS only in flower or leaf, respectively. Many heat-induced CaHsp20s, such as CaHsp16.6a, 17.6, 21.1, 21.2, 22.0, 24.3a, and 25.8 were expressed at higher level in treated pepper leaves than other three tissues, however, the highest levels of CaHsp16.3 and 22.6 were detected in flowers with HS treatment, and the expression level of CaHsp17.7a was highest in treated roots. The transcripts of 12 (34.3\%) genes (CaHsp17.8a, 17.8b, 18.4a, 18.4b, 21.7, 22.1, 24.3b, $26.5,27.6,28.4,30.1$, and 35.5$)$ were not visible in the both of the normal temperature and HS conditions.

\section{Expression Profiles of CaHsp20 Genes in Response to Heat Stress}

In order to investigate the heat response profile for CaHsp20 genes, the transcript patterns of each CaHsp20 in leaves from thermosensitive line B6 and thermotolerant line R9 with continuous heat stress treatment $\left(40^{\circ} \mathrm{C}\right.$ for $\left.4 \mathrm{~h}\right)$ were compared. As shown in Figure 7, strong response to HS in all of the 35 CaHsp20 genes from both lines (B6 and R9) were observed, in which a majority of these genes were up-regulated by HS treatment. For example CaHsp16.4, 18.7, 22.0, 25.8, and 25.9 were highly induced after a short-term $\mathrm{HS}\left(40^{\circ} \mathrm{C}\right.$ for $\left.0.5 \mathrm{~h}\right)$ and their high expression levels were maintained until the end of the HS treatment, while only CaHsp21.7 and 28.4 were down-regulated by HS in R9. Generally, the peaks of relative expression levels of CaHsp20 genes (compared with HS for $0 \mathrm{~h}$ ) in thermosensitive line B6 treated with HS were higher than those of thermotolerant line R9, however the appearance of peak values were observed at 2 or $4 \mathrm{~h}$ in B6 but at $0.5 \mathrm{~h}$ in R9 after HS treatment. In addition, it was worth noting that compared with other CaHsp20 members, CaHsp16.4, 18.7, 22.0, 25.8, and 25.9 which were significantly up-regulated in B6 leaves treated with HS, however, no main heat-response genes was detected in line R9 after HS treatment, although many CaHsp20 genes were induced by HS in R9.

\section{Analysis of Stress-related cis-elements in the CaHsp20 Promoters}

To further explore the possible regulation mechanism of CaHsp20 genes in the HS response of pepper, the cis-elements involving in the activation of defense-related genes in the promoter regions of CaHsp20s were also scanned, and the promoter regions ( $-500 \mathrm{bp}$ upstream of the translation start site) of a total of $30 \mathrm{CaHsp20}$ genes (promoter regions of CaHsp18.4a, 21.1, 21.2, 22.1, and 28.4 were absent) from PGD were used (Figure 8). One to three HSE elements (heat stress responsiveness) were found in the promoter regions of 12 genes (CaHsp16.3, 16.4, 17.6, 17.8a, 17.8b, 18.1a, 18.2a, 22.0, $25.8,25.9,26.5$, and 27.6), respectively, in which the number of HSE elements in the promoter region of CaHsp17.8b was the maximum. Although HSE elements could not be found in -500 bp promoter regions of other 11 CaHsp20 genes (CaHsp16.7, 17.2, 17.7b, 18.1b, 18.4b, 21.7, 23.8, 24.2, 24.3b, 30.1, and 35.5), 1-3 HSE elements were detected in their $-1500 \mathrm{bp}$ promoter regions, however, no any HSE element was present in either -1500 or $-500 \mathrm{bp}$ promoter regions of the rest seven CaHsp20s (CaHsp16.6a, 16.6b, 17.7a, 18.2b, 18.7, 22.6, and 24.3a). In addition, other stress-related cis-elements were detected, including TATA-boxes in all 30 selected promoter regions, CCAAT-motif in 23 genes, TC-rich repeats in seven 


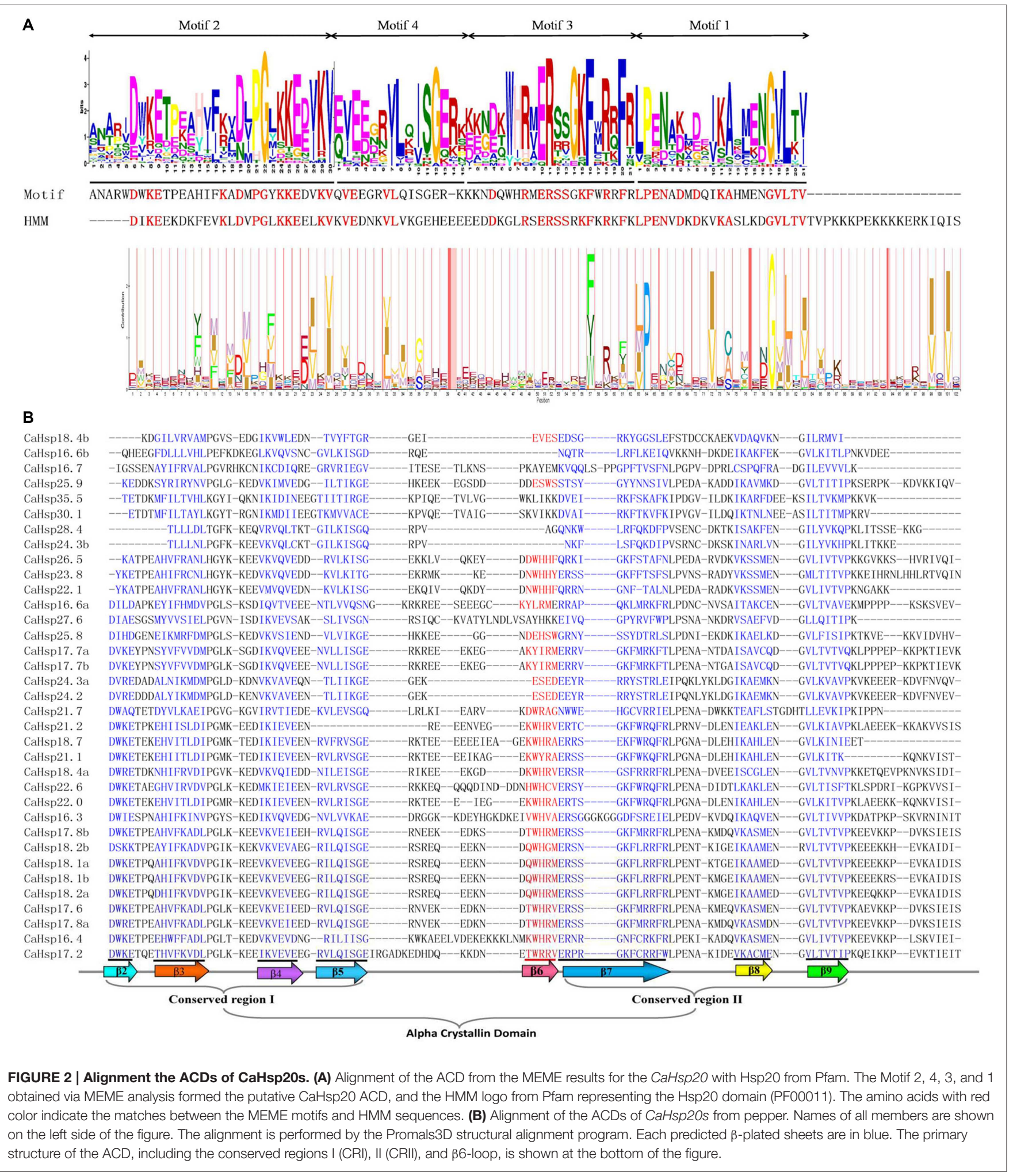




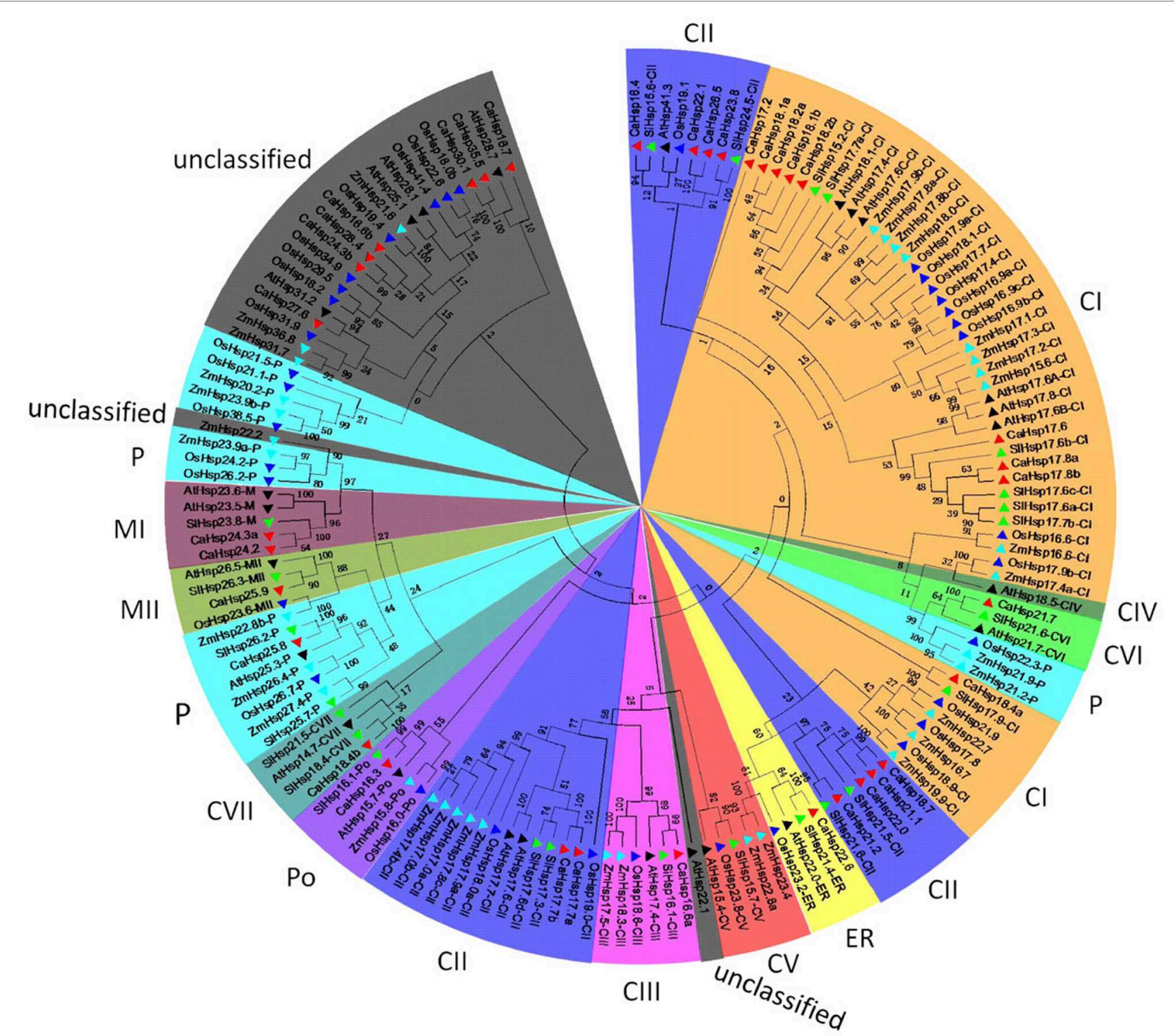

FIGURE 3 | Phylogenetic tree of pepper, tomato, and Arabidopsis Hsp20 proteins. The Hsp20 proteins are used for construction of the phylogenetic tree using the MEGA 5.10 program. Unrooted Neighbor-Joining analysis is performed with pairwise deletion and Poisson correction. For the tomato (prefixed by SI), Arabidopsis (prefixed by $A t$ ), rice (prefixed by Os), and maize (prefixed by $Z m$ ) Hsp20 proteins, both gene names and subfamily names are used, and marked with green, black, blue, and cyan triangles, respectively. CaHsp20 proteins are marked with red triangles. The 12 subfamilies are marked by different colors, and the Hsp20 proteins unclassified have been colored in gray.

genes (CaHsp17.8a, 18.1b, 18.2a, 18.2b, 18.7, 30.1, and 35.5), and W-box in three genes (CaHsp18.4b, 18.7, and 25.8).

\section{Discussion}

It has been demonstrated that Hsp20s can prevent the irreversible aggregation of denaturing proteins and enhance plant-acquired thermotolerance (Wang et al., 2004; Haslbeck and Vierling, 2015). Benefiting from the availability of whole genome sequence in recent years, several Hsp20 families have been identified from Arabidopsis, rice, soybean, and Populus trchocarpa (Waters et al.,
2008; Lopes-Caitar et al., 2013), however, very little is known about this family in pepper.

The present study describes the identification of a total of 35 CaHsp20 genes from pepper, investigation of their structure, chromosomal organization, evolutionary relationships, prediction of interaction network, and expression patterns across the different tissues and under heat stress condition, which provides a basis for comprehensively understanding the CaHsp20 gene family in pepper and makes a base for future functional divergence of the Hsp20 genes in the Solanaceae family. 


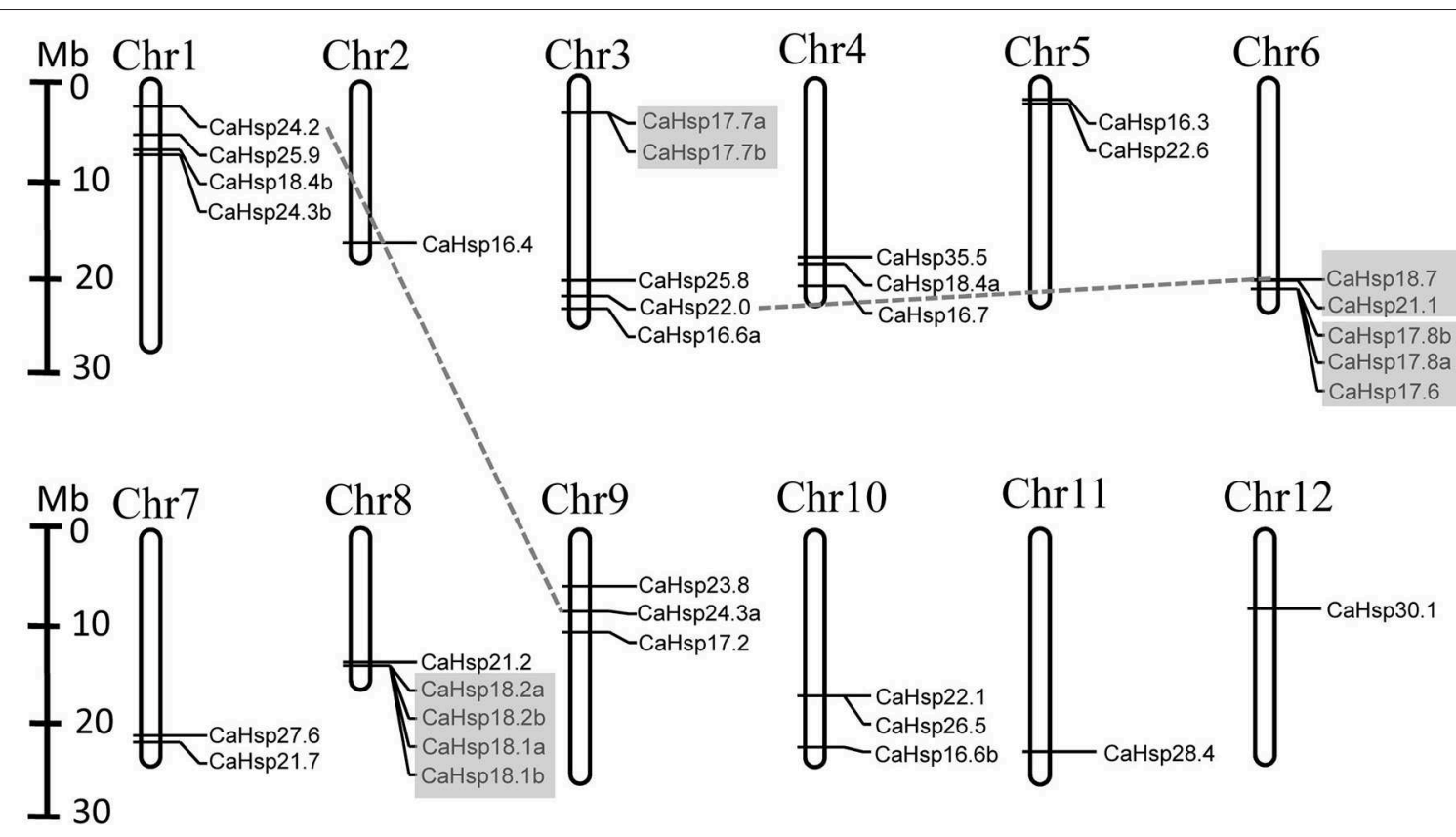

FIGURE 4 | Location and duplications of CaHsp20 paralogs on pepper chromosomes. The scale represents megabases (Mb). The chromosome numbers are indicated at the top of each bar. The CaHsp20 genes present on duplicated chromosomal segments are connected by gray dotted lines and tandem duplicated genes are marked by gray rectangles.

Although having the most diversity among the molecular chaperone families, the Hsp20s share a conserved structural domain, the ACD flanked by a non-conserved N-terminal sequence of variable length and a conserved short C-terminal sequence (Kriehuber et al., 2010; Bondino et al., 2012). Characterization and multiple sequence alignments divulged that all selected $35 \mathrm{CaHsp} 20$ s contained the ACD with various sequences. The CRI (motif 2 and 4 ) consisted of $\beta 2, \beta 3, \beta 4$, and $\beta 5$ and CRII (part of motif 3 and 1) consisted of $\beta 7, \beta 8$, and $\beta 9$ in $A C D$ were structurally conserved, containing the signature typical residues Pro-X(14)-Gly-Val-Leu and Pro-X(14)$\mathrm{X}-\mathrm{Val} / \mathrm{Leu} / \mathrm{Ile}-\mathrm{Val} / \mathrm{Leu} / \mathrm{Il}$, respectively (Figures 1, 2B; Caspers et al., 1995; Ouyang et al., 2009). Most of the CaHsp20 ACDs also contain the $\beta 6$ sheet, the non-conserved motif connecting $\beta 5$ in CRI and $\beta 7$ in CRII (Bondino et al., 2012), while it is replaced by sequences of unknown function in four CaHsp20 members (CaHsp16.7, 27.6, 30.1, and 35.5) or absent in three members (CaHsp16.6b, 24.3b, and 28.4). Plant Hsp20 proteins of CIV clusters lack the $\beta 6$ sheet (Waters, 2013) but more interesting is that none of the Hsp20 members from pepper, tomato, rice, and maize are found in the CIV cluster, while found in Arabidopsis Hsp20 family, in addition, the above seven CaHsp20 genes are not clustered into any subfamily (Figure 3 ), it can be inferred that these genes from pepper belong to CIV cluster or form a new cluster. Because the $\beta 6$ sheet is crucial for dimer formation and oliogomerization, whether these CaHsp20 genes lacking of $\beta 6$ sheet can function as a molecular chaperone still need further comparative studies.

Gene organization also plays an important role in the evolution of multiple gene families and in response to stress conditions (Xu et al., 2012). Sixteen CaHsp20 genes are intronless (pattern 1), 18 genes contain one intron (pattern 2), only one gene (CaHsp27.6) contains five introns (pattern 3; Table 1). Most CaHsp20 members of CI and ER subfamilies have no intron (pattern 1) and the members of MII subfamily have one intron (pattern 2; Figure 3; Table 1), which is consist with rice Hsp20 family, while the patterns of CaHsp20 members and Hsp2Os from rice belong to CII, Po and P subfamilies are not the same (Ouyang et al., 2009), indicating that although intron patterns shed light on the phylogenetic relationship, there is difference between different species. Most of these CaHsp20 genes are highly induced by HS in different tissues (Figure 6), which may indicate that the expression levels of genes without introns or with a short length intron will be enhanced under HS in plants (LopesCaitar et al., 2013). The compact genes have faster response to HS and protect plants from HS damage better than those genes contain many introns, and the compact genes are gradually selected by stress conditions during the long-term evolution (Jeffares et al., 2008), which may be the reason that Hsp20 family members form a first line of defense against protein aggregation caused by HS condition in order to maintain protein homeostasis and enhance the plant thermotolerance (Haslbeck and Vierling, 2015).

Gene duplication events play a significant role in expansions of gene family members and evolutionary mechanisms of genomes (Vision et al., 2000). Tandem duplications and segmental duplications are the major evolutionary patterns (Kong et al., 2007). In this analysis, $24 \mathrm{Hsp} 20$ genes were identified in tomato, however, in pepper with a genome size four-fold larger than that of tomato, only $35 \mathrm{CaHsp} 20$ genes were 


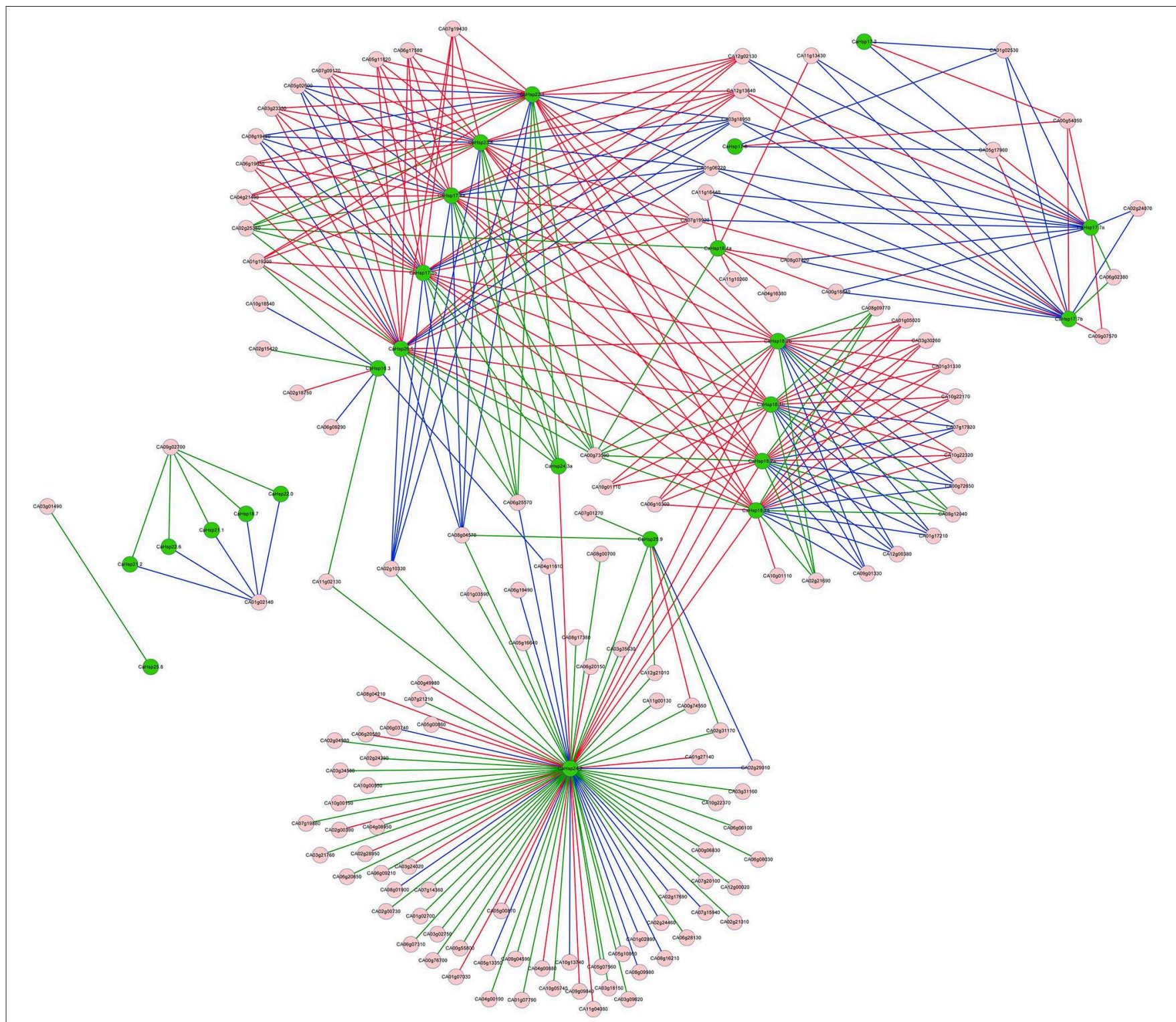

FIGURE 5 | The interaction network of CaHsp20 genes in pepper according to the orthologs in Arabidopsis. The green circles represent the CaHsp20 genes, and pink circles represent the pepper genes interaction with CaHsp20 genes. The red lines represent the PCC > 0 (Pearson Correlation Coefficient), the blue lines mean the PCC $<0$, and the green lines represent the PCC is not calculated.

found. The reason for this might be the whole-genome duplication (WGD) events in pepper and tomato (Qin et al., 2014). A total of 14 CaHsp20 duplicated genes were found in pepper chromosomes, including one segmental duplication event (CaHsp24.2/CaHsp24.3a) and three tandem duplication events (CaHsp17.7a/17.7b, CaHsp17.6/17.8a/17.8b and CaHsp18.1a/18.1b/18.2a/18.2b; Figure 4), contributing to evolution of MI, CI and CII genes. Besides, CaHsp18.7, 21.1, and 22.0 experienced one segmental duplication and one tandem duplication event, also contributing to evolution of CII genes. In previous report, the CI subfamily in plant including tomato, is always the largest (Waters, 2013), however the number of CaHsp20 members belong to CII subfamily is largest (Figure 3), which is not consistent with that of the other plants. The reason may be attributed to CaHsp20 genes of CII subfamily appeared after speciation through multiple gene duplication. Due to the opinion that gene duplication in tandem mainly occurs in the genes encoded membrane proteins and stress-related proteins, we can suggest that the dominant tandem duplications in $\mathrm{CaHsp} 20$ genes may be related to the fact that CaHsp20s are regulated by HS (Cannon et al., 2004; Rizzon et al., 2006; Hanada et al., 2008; Giorno et al., 2012).

The tissue-specific expression patterns of Hsp20 genes have been reported in various species, such as Arabidopsis, rice, and soybean, however, there is no uniform pattern of gene expression 


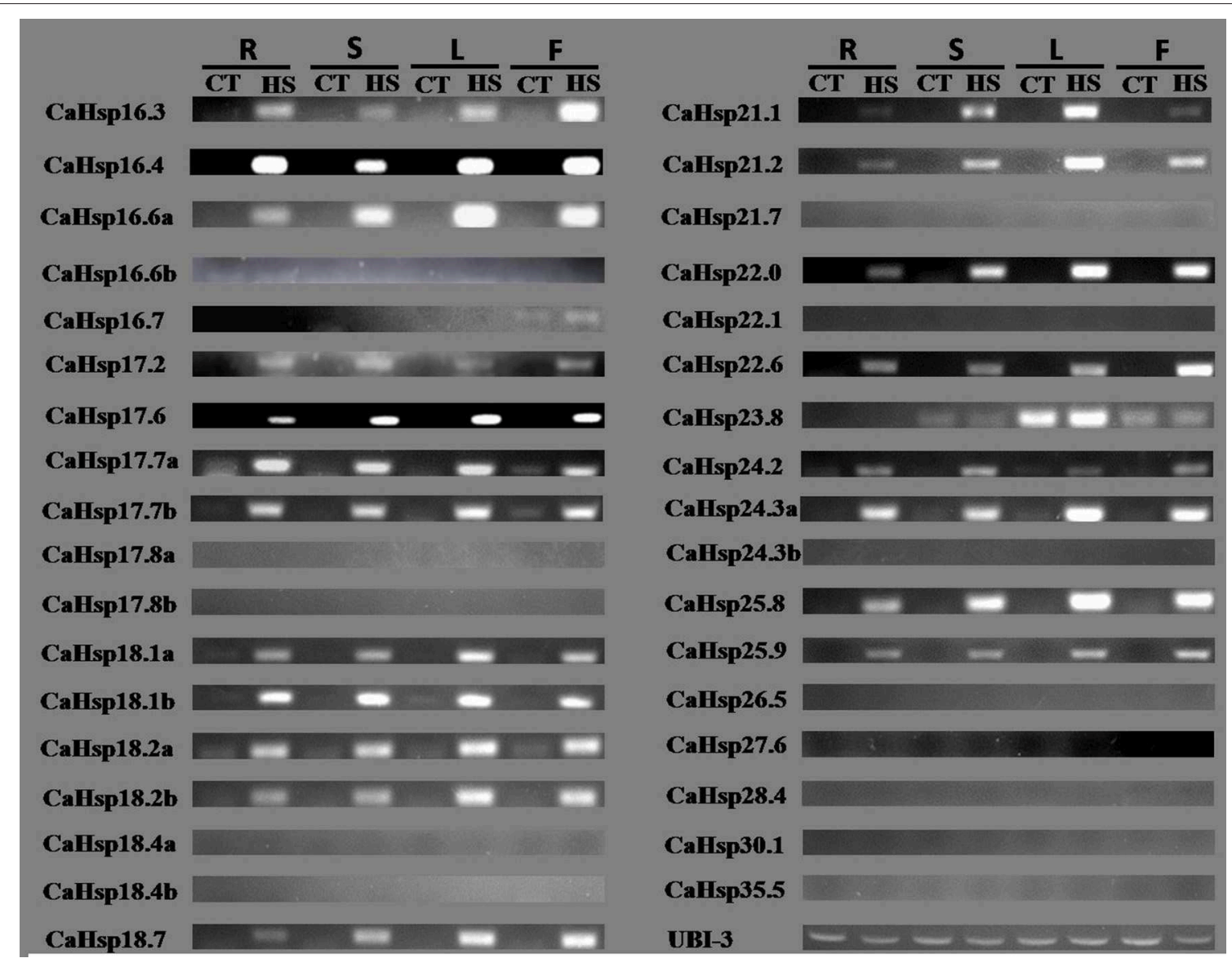

FIGURE 6 | RT-PCR analysis of expression pattern of 35 CaHsp20 genes in four pepper tissues under normal and HS conditions. R, root; S, stem; L, leaf; F, flower. Normal condition: $26^{\circ} \mathrm{C}$ for $2 \mathrm{~h}$; $\mathrm{HS}$ condition: $40^{\circ} \mathrm{C}$ for $2 \mathrm{~h}$. The pepper ubiquitin-conjugating protein gene (CaUB/-3) is used as an internal control.

for all the plant Hsp20 genes (Lopes-Caitar et al., 2013; Waters, 2013). Most CaHsp20 genes have no or low expression in the four examined tissues (root, stem, leaf, and flower) from pepper thermotolerant line R9 under normal temperature condition, but are strongly induced by HS (Figure 6), which is largely consistent with the previous reported that almost all the plant sHsps are heat induced. Among the $35 \mathrm{CaHsp} 20$ genes, $\mathrm{CaHsp} 23.8$ possesses the most distinctive expression pattern compared with other CaHsp20 genes, which expresses constitutively in stems, leaves and flowers, but almost no induction by HS. Waters (2013) reported that AtHsp21.7CV gene lacking chaperone activity from Arabidopsis is constitutively expressed in shoots and not up-regulated by HS, therefore, it can be assumed that CaHsp23.8 also may lack the chaperone activity, but it needs more scientific evidence. In addition, the expression of CaHsp16.6b, $16.7,17.7 a, 17.7 b$, and $18.2 a$ were detected in various tissues under normal condition, we can suggest that these genes exhibit specific housekeeping activities as with some Hsp20 genes from rice and soybean (Ouyang et al., 2009; Lopes-Caitar et al., 2013).

It has been demonstrated that sHsps are associated with thermotolerance in various plant species, however, many Hsp20s exist in plants which may lead to function redundancy of individual genes (Sun and MacRae, 2005; Waters, 2013). The comprehensive analysis of the expression for individual Hsp2O members under HS is necessary for further in-depth functional analyses of $H s p 20$ genes in plant thermotolerance. In this study, the dynamic expression of all 35 CaHsp20s in thermosensitive line B6 and thermotolerant line R9 with HS treatment were analyzed (Figure 7). Almost all CaHsp20 genes are induced by HS treatment in line both $\mathrm{B} 6$ and $\mathrm{R} 9$, indicating that the accumulation of the CaHsp20s effectively reduce the damage from HS and enhance pepper thermotolerance by binding denaturing proteins and preventing them from irreversible aggregation (Haslbeck and Vierling, 2015). It is well-known that $H s f s$ are activated under HS condition, and then bind the HSE elements of the promoters of the Hsp genes to regulate the expression of downstream Hsp genes. The results that the promoter regions of $\mathrm{CaHsp20}$ genes contain various ciselements (Figure 8) and CaHsp20s can interact with many functional genes (Figure 5) suggest that the expression regulation network of CaHsp20 genes under HS condition is a complex signal transduction pathway, not just $H s f s$. This hypothesis can be supported by the observation that some CaHsp20 genes (CaHsp16.6a, 17.7a, 18.7, 21.2, 22.6, and 24.3) whose promoter regions $(-1500 \mathrm{bp})$ do not contain the HSEs can be highly 


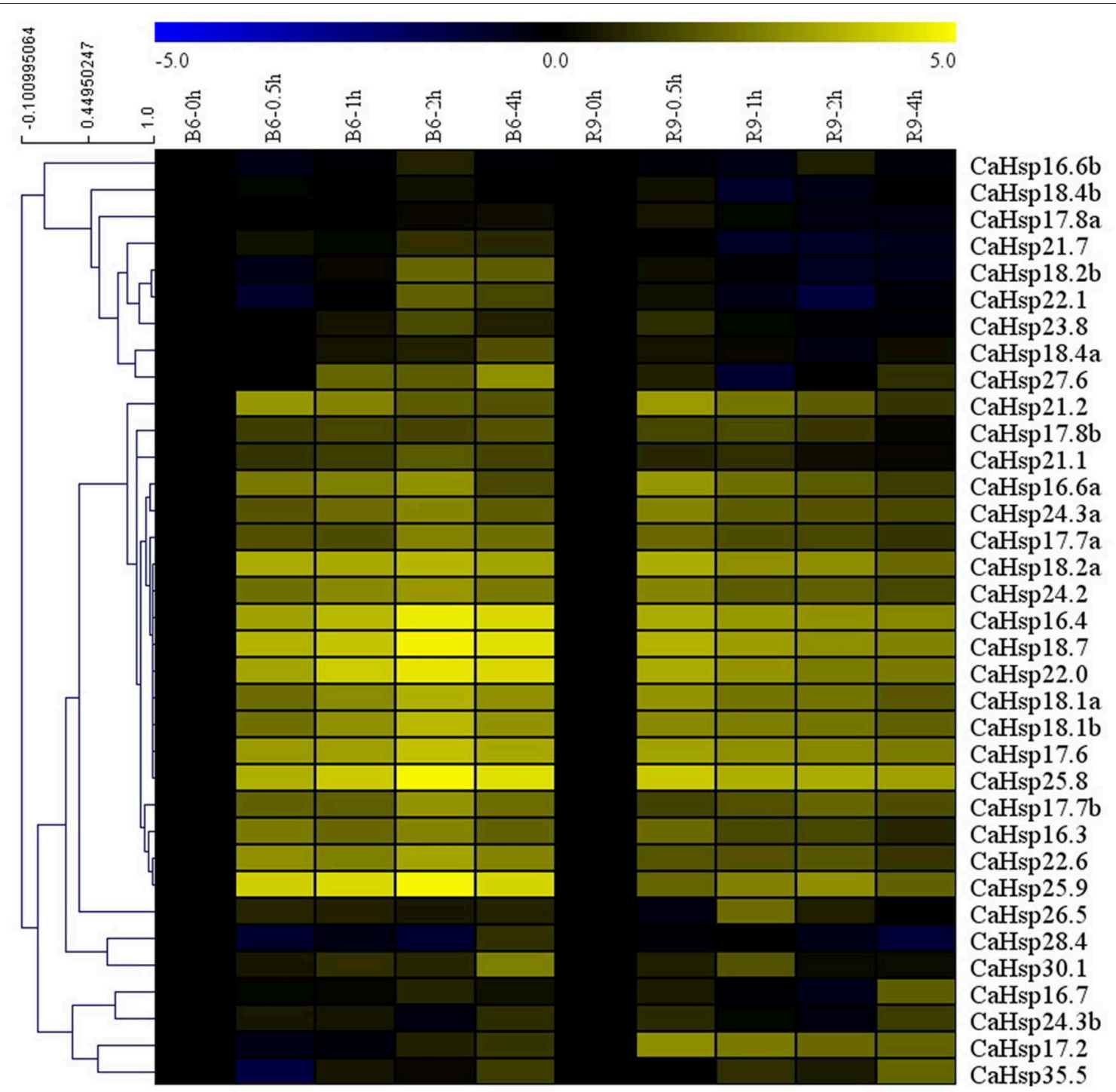

FIGURE 7 | Heat map showing CaHsp20 genes expression pattern in pepper thermosensitive line B6 and thermotolerant line R9 under HS condition. Leaves of B6 and R9 seedlings (6-8 true leaves) are used to test the changes of CaHsp2O genes expression level at different time points (0, 0.5, 1, 2, and 4 h) with HS treatment $\left(40^{\circ} \mathrm{C}\right)$. CaUBl-3 is used as an internal control. qRT-PCR data are shown relative to $0 \mathrm{~h}$. The relative expression levels were calculated using the $2^{-\Delta \Delta \mathrm{CT}}$ method, and normalized using log10. The heat map was created using MeV.

induced by HS treatment. Intriguingly, CaHsp17.7a and $17.7 b$ genes are in a tandem duplication share a similar expression pattern in both lines B6 and R9 with HS treatment, however, another tandem duplication pair CaHsp18.2a and $18.2 b$ exhibit different expression patterns in both lines B6 and R9, so it seems that not all the members of the same sHsp subfamily have the same expression patterns (Waters, 2013). It is worth noting that the peak of the expression of most CaHsp20 genes in R9 (HS for 0.5 or $1 \mathrm{~h}$ ) appeared earlier than in B6 (HS for 2 or $4 \mathrm{~h}$ ), which may indicate that when suffering from HS, the pepper thermotolerant line R9 can start the thermal response mechanism faster than the thermosensitive line B6, however, the relationship between the function of CaHsp20 genes and thermotolerant mechanism in pepper need to be further studied.

\section{Conclusions}

In this study, a genome-wide analysis of CaHsp20 gene family in pepper was performed to reveal gene structure, phylogenetic relationship, evolution, and expression profiles in different tissues (root, stem, leaf, and flower) and HS conditions. Most CaHsp20 genes maintained the low expression level under normal temperature condition and could be highly induced by HS, indicating that they played a key role in the process of pepper acquired thermotolerance. The data in this study will provide a foundation for functional characterization of CaHsp20 family members, and make a contribution toward illuminating the mechanism of pepper acquired thermotolerance, and even the adaptability of pepper to other stress conditions. 


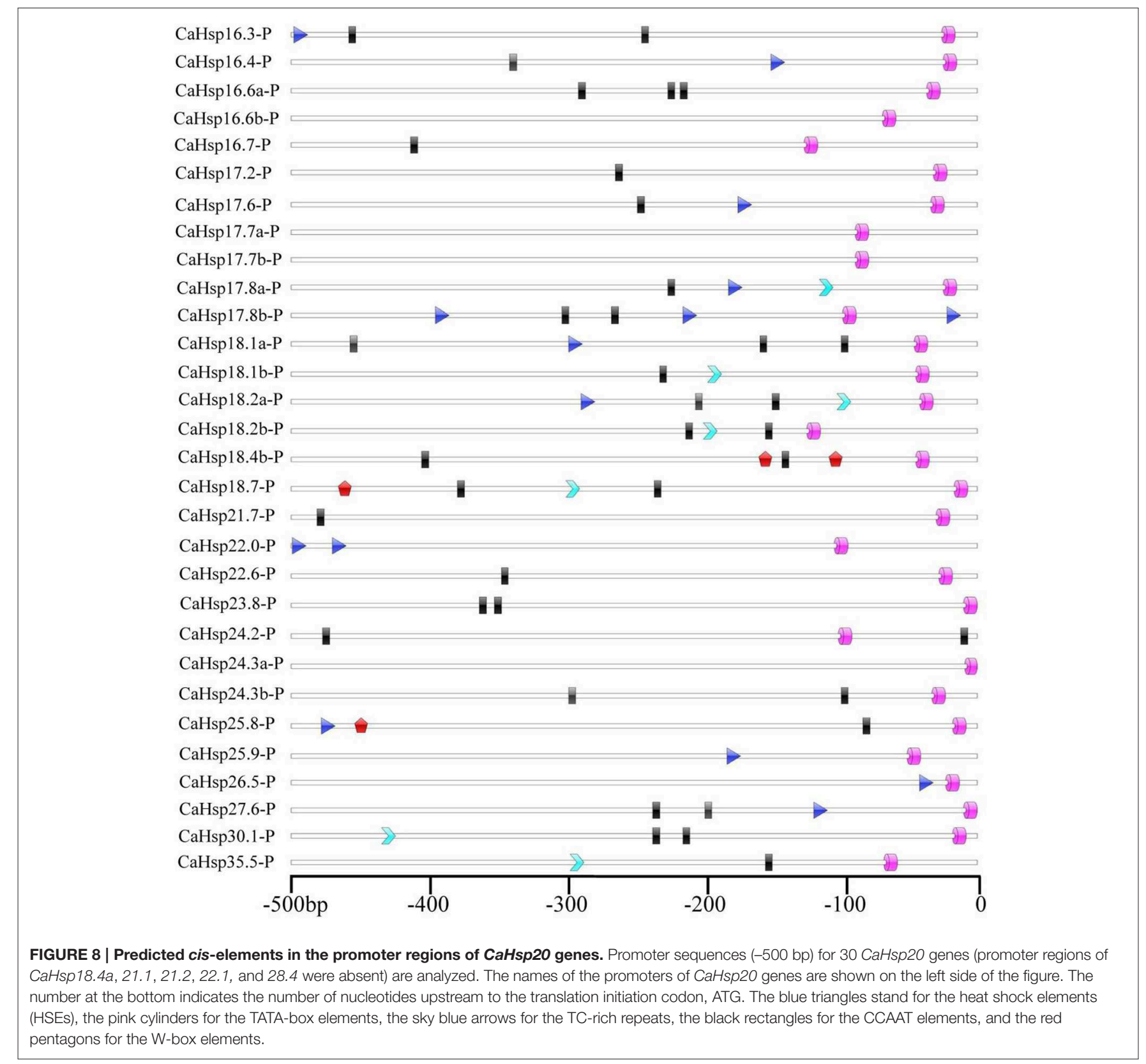

\section{Acknowledgments}

This work was supported by the National Natural Science Foundation of China (Grant No. 31272163, 31572114), the Shaanxi Agriculture Science and Technology Projects (Grant No. 2014K01-14-01), Jiangsu Agriculture Science and Technology Innovation Fund [CX(12)1004], the Opening Fund of Key Laboratory for Crop Biotechnology of Xinjiang Uygur Autonomous Region (Grant No. XJYS0302-2014-03) and the Tang Zhongying Fund for Breeding of Northwest A\&F University.

\section{Supplementary Material}

The Supplementary Material for this article can be found online at: http://journal.frontiersin.org/article/10.3389/fpls.2015. 00806

Figure S1 | The logic flowchart of identification of pepper Hsp20 genes.

Table S1 | The gene IDs of Hsp20 members from Arabidopsis, tomato, maize and rice.

Table S2 | Primer sequences used for semi-quantitative RT-PCR and quantitative real-time PCR analysis. 


\section{References}

Basha, E., Friedrich, K. L., and Vierling, E. (2006). The N-terminal arm of small heat shock proteins is important for both chaperone activity and substrate specificity. J. Biol. Chem. 281, 39943-39952. doi: 10.1074/jbc.M607677200

Bondino, H. G., Valle, E. M., and Ten Have, A. (2012). Evolution and functional diversification of the small heat shock protein/ $\alpha$-crystallin family in higher plants. Planta 235, 1299-1313. doi: 10.1007/s00425-011-1575-9

Cannon, S. B., Mitra, A., Baumgarten, A., Young, N. D., and May, G. (2004). The roles of segmental and tandem gene duplication in the evolution of large gene families in Arabidopsis thaliana. BMC Plant Biol. 4:10. doi:10.1186/1471-22294-10

Caspers, G. J., Leunissen, J. A., and de Jong, W. W. (1995). The expanding small heat-shock protein family, and structure predictions of the conserved "alpha-crystallin domain". J. Mol. Evol. 40, 238-248. doi: 10.1007/BF00163229

Charng, Y. Y., Liu, H. C., Liu, N. Y., Hsu, F. C., and Ko, S. S. (2006). Arabidopsis Hsa32, a novel heat shock protein, is essential for acquired thermotolerance during long recovery after acclimation. Plant Physiol. 140, 1297-1305. doi: 10.1104/pp.105.074898

Emanuelsson, O., Nielsen, H., Brunak, S., and von Heijne, G. (2000). Predicting subcellular localization of proteins based on their $\mathrm{N}$-terminal amino acid sequence. J. Mol. Biol. 300, 1005-1016. doi: 10.1006/jmbi.2000.3903

Giese, K. C., and Vierling, E. (2004). Mutants in a small heat shock protein that affect the oligomeric state. Analysis and allele-specific suppression. J. Biol. Chem. 279, 32674-32683. doi: 10.1074/jbc.M404455200

Giorno, F., Guerriero, G., Baric, S., and Mariani, C. (2012). Heat shock transcriptional factors in Malusdomestica: identification, classification and expression analysis. BMC Genomics 13:639. doi: 10.1186/1471-2164-13-639

Giorno, F., Wolters-Arts, M., Grillo, S., Scharf, K. D., Vriezen, W. H., and Mariani, C. (2010). Developmental and heat stress-regulated expression of HsfA2 and small heat shock proteins in tomato anthers. J. Exp. Bot. 61, 453-462. doi: $10.1093 /$ jxb/erp316

Glazebrook, J. (2001). Genes controlling expression of defense responses in Arabidopsis-2001 status. Curr. Opin. Plant Biol. 4, 301-308. doi: 10.1016/S1369-5266(00)00177-1

Gu, Z., Cavalcanti, A., Chen, F. C., Bouman, P., and Li, W. H. (2002). Extent of gene duplication in the genomes of Drosophila, nematode, and yeast. Mol. Biol. Evol. 19, 256-262. doi: 10.1093/oxfordjournals.molbev.a004079

Guo, M., Zhai, Y. F., Lu, J. P., Chai, L., Chai, W. G., Gong, Z. H., et al. (2014). Characterization of CaHsp70-1, a pepper heat-shock protein gene in response to heat stress and some regulation exogenous substances in Capsicum annuum L. Int. J. Mol. Sci. 15, 19741-19759. doi: 10.3390/ijms151119741

Guo, S. J., Zhou, H. Y., Zhang, X. S., Li, X. G., and Meng, Q. W. (2007). Overexpression of CaHSP26 in transgenic tobacco alleviates photoinhibition of PSII and PSI during chilling stress under low irradiance. J. Plant Physiol. 164, 126-136. doi: 10.1016/j.jplph.2006.01.004

Hanada, K., Zou, C., Lehti-Shiu, M. D., Shinozaki, K., and Shiu, S. H. (2008). Importance of lineage-specific expansion of plant tandem duplicates in the adaptive response to environmental stimuli. Plant Physiol. 148, 993-1003. doi: 10.1104/pp.108.122457

Haslbeck, M., and Vierling, E. (2015). A first line of stress defense: small heat shock proteins and their function in protein homeostasis. J. Mol. Biol. 427, 1537-1548. doi: 10.1016/j.jmb.2015.02.002

Higo, K., Ugawa, Y., Iwamoto, M., and Korenaga, T. (1999). Plant cis-acting regulatory DNA elements (PLACE) database: 1999. Nucleic Acids Res. 27, 297-300. doi: 10.1093/nar/27.1.297

Jaya, N., Garcia, V., and Vierling, E. (2009). Substrate binding site flexibility of the small heat shock protein molecular chaperones. Proc. Natl. Acad. Sci. U.S.A. 106, 15604-15609. doi: 10.1073/pnas.0902177106

Jeffares, D. C., Penkett, C. J., and Bähler, J. (2008). Rapidly regulated genes are intron poor. Trends Genet. 24, 375-378. doi: 10.1016/j.tig.2008.05.006

Kim, S., Park, M., Yeom, S. I., Kim, Y. M., Lee, J. M., Lee, H. A., et al. (2014). Genome sequence of the hot pepper provides insights into the evolution of pungency in Capsicum species. Nat. Genet. 46, 270-278. doi: 10.1038/ng.2877

Kirschner, M., Winkelhaus, S., Thierfelder, J. M., and Nover, L. (2000). Transient expression and heat-stress-induced co-aggregation of endogenous and heterologous small heat-stress proteins in tobacco protoplasts. Plant J. 24, 397-411. doi: 10.1046/j.1365-313x.2000.00887.x
Kong, H., Landherr, L. L., Frohlich, M. W., Leebens-Mack, J., Ma, H., and dePamphilis, C. W. (2007). Patterns of gene duplication in the plant SKP1 gene family in angiosperms: evidence for multiple mechanisms of rapid gene birth. Plant J. 50, 873-885. doi: 10.1111/j.1365-313X.2007.03097.x

Kriehuber, T., Rattei, T., Weinmaier, T., Bepperling, A., Haslbeck, M., and Buchner, J. (2010). Independent evolution of the core domain and its flanking sequences in small heat shock proteins. FASEB J. 24, 3633-3642. doi: 10.1096/fj.10-156992

Lescot, M., Déhais, P., Thijs, G., Marchal, K., Moreau, Y., van de Peer, Y., et al. (2002). PlantCARE, a database of plant cis-acting regulatory elements and a portal to tools for in silico analysis of promoter sequences. Nucleic Acids Res. 30, 325-327. doi: 10.1093/nar/30.1.325

Liu, R. H., and Meng, J. L. (2003). MapDraw: a microsoft excel macro for drawing genetic linkage maps based on given genetic linkage data. Hereditas 25, 317-321. doi: 10.3321/j.issn:0253-9772.2003.03.019

Lopes-Caitar, V. S., de Carvalho, M. C., Darben, L. M., Kuwahara, M. K., Nepomuceno, A. L., Dias, W. P., et al. (2013). Genome-wide analysis of the Hsp20 gene family in soybean: comprehensive sequence, genomic organization and expression profile analysis under abiotic and biotic stresses. BMC Genomics 14:577. doi: 10.1186/1471-2164-14-577

Mittler, R. (2006). Abiotic stress, the field environment and stress combination. Trends Plant Sci. 11, 15-19. doi: 10.1016/j.tplants.2005.11.002

Mittler, R., Finka, A., and Goloubinoff, P. (2012). How do plants feel the heat? Trends Biochem. Sci. 37, 118-125. doi: 10.1016/j.tibs.2011.11.007

Ouyang, Y., Chen, J., Xie, W., Wang, L., and Zhang, Q. (2009). Comprehensive sequence and expression profile analysis of $\mathrm{Hsp} 20$ gene family in rice. Plant Mol. Biol. 70, 341-357. doi: 10.1007/s11103-009-9477-y

Qin, C., Yu, C., Shen, Y., Fang, X., Chen, L., Min, J., et al. (2014). Whole-genome sequencing of cultivated and wild peppers provides insights into Capsicum domestication and specialization. Proc. Natl. Acad. Sci. U.S.A. 111, 5135-5140. doi: 10.1073/pnas.1400975111

Rizzon, C., Ponger, L., and Gaut, B. S. (2006). Striking similarities in the genomic distribution of tandemly arrayed genes in Arabidopsis and rice. PLoS Comput. Biol. 2:e115. doi: 10.1371/journal.pcbi.0020115

Sarkar, N. K., Kim, Y. K., and Grover, A. (2009). Rice sHsp genes: genomic organization and expression profiling under stress and development. $B M C$ Genomics 10:393. doi: 10.1186/1471-2164-10-393

Scharf, K. D., Berberich, T., Ebersberger, I., and Nover, L. (2012). The plant heat stress transcription factor (Hsf) family: structure, function and evolution. Biochim. Biophys. Acta 1819, 104-119. doi: 10.1016/j.bbagrm.2011.10.002

Scharf, K. D., Siddique, M., and Vierling, E. (2001). The expanding family of Arabidopsis thaliana small heat stress proteins and a new family of proteins containing alpha-crystallin domains (Acd proteins). Cell Stress Chaperones 6, 225-237. doi: 10.1379/1466-1268(2001)006<0225:TEFOAT > 2.0.CO;2

Siddique, M., Gernhard, S., von Koskull-Döring, P., Vierling, E., and Scharf, K. D. (2008). The plant sHSP superfamily: five new members in Arabidopsis thaliana with unexpected properties. Cell Stress Chaperones 13, 183-197. doi: 10.1007/s12192-008-0032-6

Schmittgen, T. D., and Livak, K. J. (2008). Analyzing real-time PCR data by the comparative $C_{T}$ method. Nat. Protoc. 3, 1101-1108. doi: 10.1038/nprot. 2008.73

Sun, W., van Montagu, M., and Verbruggen, N. (2002). Small heat shock proteins and stress tolerance in plants. Biochim. Biophys. Acta 1577, 1-9. doi: 10.1016/S0167-4781(02)00417-7

Sun, Y., and MacRae, T. H. (2005). Small heat shock proteins: molecular structure and chaperone function. Cell. Mol. Life Sci. 62, 2460-2476. doi: 10.1007/s00018005-5190-4

Sung, D. Y., Kaplan, F., Lee, K. J., and Guy, C. L. (2003). Acquired tolerance to temperature extremes. Trends Plant Sci. 8, 179-187. doi: 10.1016/S13601385(03)00047-5

Tamura, K., Peterson, D., Peterson, N., Stecher, G., Nei, M., and Kumar, S. (2011). MEGA5: molecular evolutionary genetics analysis using maximum likelihood, evolutionary distance, and maximum parsimony methods. Mol. Biol. Evol. 28, 2731-2739. doi: 10.1093/molbev/msr121

Vierling, E. (1991). The roles of heat shock proteins in plants. Annu. Rev. Plant Biol. 42, 579-620. doi: 10.1146/annurev.pp.42.060191.003051

Vision, T. J., Brown, D. G., and Tanksley, S. D. (2000). The origins of genomic duplications in Arabidopsis. Science 290, 2114-2117. doi: $10.1126 /$ science.290.5499.2114 
Wan, H., Yuan, W., Ruan, M., Ye, Q., Wang, R., Li, Z., et al. (2011). Identification of reference genes for reverse transcription quantitative real-time PCR normalization in pepper (Capsicum annuumL.). Biochem. Biophys. Res. Commun. 416, 24-30. doi: 10.1016/j.bbrc.2011.10.105

Wang, L., Guo, K., Li, Y., Tu, Y., Hu, H., Wang, B., et al. (2010). Expression profiling and integrative analysis of the CESA/CSL superfamily in rice. BMC Plant Biol. 10:282. doi: 10.1186/1471-2229-10-282

Wang, W., Vinocur, B., and Altman, A. (2003). Plant responses to drought, salinity and extreme temperatures: towards genetic engineering for stress tolerance. Planta 218, 1-14. doi: 10.1007/s00425-003-1105-5

Wang, W., Vinocur, B., Shoseyov, O., and Altman, A. (2004). Role of plant heatshock proteins and molecular chaperones in the abiotic stress response. Trends Plant Sci. 9, 244-252. doi: 10.1016/j.tplants.2004.03.006

Waters, E. R. (2013). The evolution, function, structure, and expression of the plant sHSPs. J. Exp. Bot. 64, 391-403. doi: 10.1093/jxb/ ers355

Waters, E. R., Aevermann, B. D., and Sanders-Reed, Z. (2008). Comparative analysis of the small heat shock proteins in three angiosperm genomes identifies new subfamilies and reveals diverse evolutionary patterns. Cell Stress Chaperones 13, 127-142. doi: 10.1007/s12192-0080023-7

Waters, E. R., Lee, G. J., and Vierling, E. (1996). Evolution, structure and function of the small heat shock proteins in plants. J. Exp. Bot. 47, 325-338. doi: $10.1093 / \mathrm{jxb} / 47.3 .325$
Wei, F., Coe, E., Nelson, W., Bharti, A. K., Engler, F., Butler, E., et al. (2007) Physical and genetic structure of the maize genome reflects its complex evolutionary history. PLoS Genet. 3:e123. doi: 10.1371/journal.pgen.0030123

Xu, G., Guo, C., Shan, H., and Kong, H. (2012). Divergence of duplicate genes in exon-intron structure. Proc. Natl. Acad. Sci. U.S.A. 109, 1187-1192. doi: 10.1073/pnas.1109047109

Yang, S., Zhang, X., Yue, J. X., Tian, D., and Chen, J. Q. (2008). Recent duplications dominate NBS-encoding gene expansion in two woody species. Mol. Genet. Genomics 280, 187-198. doi: 10.1007/s00438-008-0355-0

Zhu, W., Lu, M. H., Gong, Z. H., and Chen, R. G. (2011). Cloning and expression of a small heat shock protein gene CaHSP24 from pepper under abiotic stress. Afr. J. Biotechnol. 10, 4968-4976. doi: 10.5897/AJB11.118

Conflict of Interest Statement: The authors declare that the research was conducted in the absence of any commercial or financial relationships that could be construed as a potential conflict of interest.

Copyright (c) 2015 Guo, Liu, Lu, Zhai, Wang, Gong, Wang and Lu. This is an open-access article distributed under the terms of the Creative Commons Attribution License (CC BY). The use, distribution or reproduction in other forums is permitted, provided the original author(s) or licensor are credited and that the original publication in this journal is cited, in accordance with accepted academic practice. No use, distribution or reproduction is permitted which does not comply with these terms. 